\title{
Decentralized Model Predictive Control of Voltage Source Converters for AC Frequency Containment
}

\author{
Lampros Papangelis $^{\mathrm{a}}$, Marie-Sophie Debry ${ }^{\mathrm{b}}$, Thibault Prevost ${ }^{\mathrm{b}}$, Patrick Panciatici ${ }^{\mathrm{b}}$, Thierry Van Cutsem $^{\mathrm{c}, \mathrm{a}}$ \\ ${ }^{a}$ Dept. of Electrical Eng. and Computer Sc., University of Liège, Belgium \\ ${ }^{b}$ Research and Development Dept. of RTE, Versailles, France \\ ${ }^{c}$ Fund for Scientific Research (FNRS), Belgium
}

\begin{abstract}
This paper presents a novel control scheme for exchange of frequency support between asynchronous AC systems through a High Voltage Direct Current link or grid. The proposed controller bears the spirit of an emergency scheme. Using only locally available measurements, each converter can identify emergency situations that could potentially lead to unacceptable frequency values. Then appropriate control actions are taken to restrain the frequency decline and prevent it from reaching the thresholds of load shedding relays. Inspired of Model Predictive Control, the method uses simplified models of the AC and DC sides of the converter, and can incorporate various constraints. The effectiveness of the method is demonstrated on a test system consisting of two asynchronous AC areas interconnected through a five-terminal HVDC grid.
\end{abstract}

Keywords: Voltage Source Converter, frequency support, Model Predictive Control, Multi-Terminal DC grid, point-to-point HVDC link

\section{Introduction}

High Voltage DC (HVDC) grids are contemplated as possible power system infrastructures to facilitate bulk power transfer over long distances and integration of distant renewable energy sources. To this purpose, apart from several point-to-point HVDC links already developed or planned, Multi-Terminal DC (MTDC) grid projects have been also proposed, such as the European Supergrid [1] and the North Sea Super Grid [2]. In order to improve the security of the resulting combined AC/DC grids, ancillary services will have to be provided by the HVDC grids to their adjacent $\mathrm{AC}$ systems, such as frequency and reactive power support [3].

HVDC grids act as "firewalls", thus the frequencies of two areas interconnected through an HVDC grid are independent and the power plants of one area do not respond to a frequency deviation in another. However, the decommissioning of conventional power plants in favor of converter-interfaced sources of energy, such as wind turbines and solar units, challenge the ability of the system to contain the frequency between acceptable limits following large disturbances [4]. To tackle this, HVDC grids should also accommodate frequency support services to

Email addresses: 1.papangelis@uliege.be. (Lampros Papangelis), marie-sophie.debry@rte-france.com (Marie-Sophie Debry), thibault.prevost@rte-france.com (Thibault Prevost), patrick.panciatici@rte-france.com (Patrick Panciatici), t.vancutsem@uliege.be (Thierry Van Cutsem) the neighboring $\mathrm{AC}$ areas. This can be achieved by providing the Voltage Source Converters (VSC) with dedicated controllers, which can adjust the power transfer in response to frequency deviations and, subsequently, enable sharing of the primary reserves of the various connected AC sub-systems [3].

Frequency support through HVDC grids has been the subject of various publications and a review of the state of the art has been reported in [5]. In most of them, a supplementary droop control is added to the control structure of the VSC, enabling it to react to frequency deviations $[6,7,8,9]$ by adjusting the power exchange with the AC system. The same concept was expanded in [10] for MTDC grids with Modular Multilevel Converters (MMC). A variant of the droop scheme was proposed in [2], where different values of droop are used depending on the severity of the disturbance. Other works have focused on improving the efficiency of the droop-based control. Reference [11] demonstrated the strong interaction of the simple frequency droop control with its DC voltage droop counterpart and proposed a method to re-tune the frequency droop gain to achieve the desired participation to frequency support. This was also addressed in [12, 13] with Receding Horizon Control taking into account DC voltage and power constraints.

A number of publications are devoted to control strategies enabling primary and inertia response by offshore wind farms connected to the main onshore grid through an HVDC grid [14, 15, 16, 17]. In this application, the main idea is to enable the offshore converters to change the 
frequency (or the $\mathrm{AC}$ voltage magnitude) they impose on the offshore grid [18]. This in turn triggers the controllers of the offshore wind turbines, which modify their active power production to provide inertial or primary frequency support. An alternative method based on directly communicating the onshore frequency deviation to the offshore wind farm was proposed in [19].

A method for inertia emulation by VSCs has been proposed in [20]. However, it is mentioned that it can be used only for inertia emulation and not for sharing of primary reserves between two asynchronous AC areas.

In general, there are two options to consider for frequency support through HVDC grids: (i) a continuously active regulation, and (ii) activation only after large disturbances. In the first option, each VSC adjusts its power exchange with the $\mathrm{AC}$ grid in response to the frequency deviation resulting in a partial coupling between the balancing controls of the various AC systems, originally decoupled. Reference [21] has also investigated the option of reaching a "frequency consensus" between the areas. In contrast, the second option considers frequency support by the VSCs as an emergency control scheme, inactive for small deviations around its nominal value [22]. Frequency support is usually activated when unusually large frequency deviations or Rates of Change of Frequency (ROCOF) are detected.

Despite the various implementation differences of the aforementioned methods for primary frequency support, the main idea remains the same: A frequency droop gain has to be selected relating the power of the VSC with the $\mathrm{AC}$ frequency deviation, sometimes complemented by an inertia emulation gain to provide some derivative response. This is the current practice in AC systems for primary frequency control by power plants, where the droop method has been the norm for several decades. However, although the droop control has proven indispensable for continuous regulation of frequency by the conventional units, the same does not necessarily hold true for the VSCs. In fact, simply specifying a frequency droop gain prevents the VSC from utilizing its maximum capacity in emergency cases, e.g. when an Under-Frequency Load Shedding (UFLS) threshold is approached. Therefore, there is a need for a more adaptive control scheme that will provide as much support as possible in stressed situations.

This paper explores a novel possibility for provision of frequency support by VSCs refraining from the requirement to select a frequency droop gain, and exploiting the almost instantaneous response of VSCs. The proposed method is activated as the last resort before the triggering of UFLS relays. It is inspired of Model Predictive Control (MPC), an optimization-based discrete-time control scheme, due to its ability to handle constraints, predict the system behavior and anticipate limit violations [23, 24]. The paper focuses on MTDC grids, however, the proposed control can be also applied on point-to-point HVDC links with small adjustments.

The rest of the paper is organized as follows. First,
Section 2 recalls some basics of VSC control. Section 3 details the proposed control. Simulation results are presented in Section 4. Finally, concluding remarks and future extensions are discussed in Section 5.

\section{Overview of VSC control basics}

This section briefly recalls some basics of VSC control with emphasis on the DC voltage droop technique.

Controlling the DC voltages is important for the secure operation of an HVDC grid. Power imbalances must be rapidly corrected, given the relatively small amount of energy stored in DC capacitors. Several methods have been proposed to this purpose, of which the DC voltage droop technique has received significant attention for MTDC grids, e.g. [25, 26, 27, 28], and has been adopted in this work. This method, inspired of AC frequency control practice, allows multiple converters to share a power imbalance in the MTDC grid while ensuring redundancy against the outage of one of them. In a droop-controlled MTDC grid some of the VSCs obey a $P-V$ characteristic defined by a power setpoint $P^{\text {set }}$, a voltage setpoint $V^{\text {set }}$ and a droop $K_{V}$. In steady state the VSC power $P$ is linked to the DC voltage $V$ through:

$$
P=P^{s e t}-K_{V}\left(V-V^{s e t}\right)
$$

where a positive power corresponds to flow from the $\mathrm{AC}$ grid towards the DC grid. Therefore, following a power deficit in the MTDC grid, the DC voltage will start decreasing and the VSC will increase the power it injects into the DC grid until the balance is restored.

A simplified diagram of the VSC control structure based on [25] is shown in Fig. 1, including the DC voltage droop control. The diagram focuses on the active power control loop, which provides the active power command $P^{c m d}$ to the current controller of the VSC. A simple open loop without a PI controller is used for the DC voltage droop control, similar to the one in [25], but alternative control schemes are possible, as discussed in [27]. The current controller adjusts the internal $\mathrm{AC}$ voltage of the $\mathrm{VSC}$ in order to inject the required currents in the AC grid. A Phase Lock Loop (PLL) is usually used to synchronize the VSC to the AC grid, also providing a measurement of the local AC frequency. Generally, there is a clear decoupling between the various control levels shown in Fig. 1. The modulation level is the fastest with a response time of some $\mu \mathrm{s}$. The response time of the current controllers and the PLL is usually in the range of some ms with the outer loops being 5-10 times slower.

Finally, the output of the "MPC controller" block is used to change the power setpoint of the VSC in response to large frequency deviations. Its operation is further described in Section 3. 


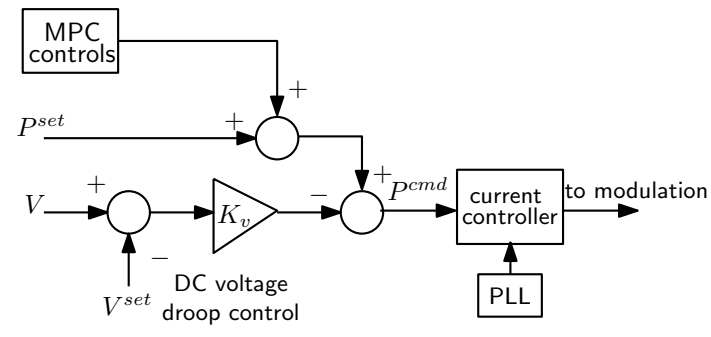

Figure 1: Simplified diagram of the VSC control structure

\section{Proposed control}

\subsection{Overall controller description}

This work treats frequency support as an "emergency" control scheme, as also suggested in [12, 22]. Therefore, for harmless frequency deviations the frequency support scheme remains inactive. This serves the purpose of preventing continuous interactions between the frequency controls of AC systems which were otherwise planned to operate asynchronously. On the other hand, in response to a large enough frequency deviation in an AC area, the VSCs connected to it correspondingly adjust the power transfer through the MTDC grid, benefiting from the primary reserves of the other AC areas.

As stated in the Introduction, this method does not require pre-defining a desired participation to frequency support. Instead, the main idea is to provide as much power as required to prevent the triggering of UFLS relays or at least reduce the amount of load shedding. Typically, these relays have multiple shedding steps with various frequency thresholds. For example, in [29] UFLS schemes with a maximum of ten shedding steps between 49 and $48 \mathrm{~Hz}$ are described. Therefore, prevention of UFLS can be translated as a constraint to keep frequency above the first frequency threshold of the relays. To achieve this, the power of each participating VSC has to be adapted when such a violation is predicted, by changing appropriately the VSC power setpoint $P^{s e t}$ of its $P-V$ characteristic (1).

Clearly, the added control should not jeopardize the operation of the MTDC grid as well the other AC areas. This imposes to obey constraints on the DC voltage, on the rate of change of powers, etc.

Finally, it is highly desirable to rely only on local measurements readily available to each VSC. By so doing, fast and reliable performance can be achieved without resorting to communication between converters, which can be subject to delays and failures.

\section{2. $M P C$ principle}

The proposed control relies on the MPC concept. This optimization-based, multi-step control scheme consists of computing a sequence of control changes which minimizes an objective and satisfies constraints in the future [23]. This optimization relies on a model of the future system evolution. In this work, two models are required: (i) a model for the DC side variables and (ii) a model of the
$\mathrm{AC}$ system frequency evolution. Both are detailed in the next sub-sections.

The MPC logic can be summarized as follows. At the current discrete time $k$, the controller has received the latest available measurements and has computed optimal control actions $\left(\Delta \mathbf{u}(k), \ldots, \Delta \mathbf{u}\left(k+N_{c}-1\right)\right)$ that should be applied from $k$ up to the end of the control horizon $k+N_{c}-1$, so that the system meets a desired target at the end of the prediction horizon $k+N_{p}\left(N_{p} \geq N_{c}\right)$. Out of this sequence, only the first component $\Delta \mathbf{u}(k)$ is applied. Then, at the next time instant $k+1$, the procedure is repeated for the updated control and prediction horizons, using the newly received measurements.

\section{3. $M T D C$ grid model}

As already mentioned, the DC voltage droop control is used to maintain the balance of the MTDC grid. By assuming that the MTDC grid dynamics are almost instantaneous, a steady-state model of the MTDC grid can be used to predict the DC voltage $(V)$ and power $(P)$ changes in response to the calculated control actions $\left(\Delta P^{s e t}\right)$ : for $j=1, \ldots, N_{c}$ :

$$
\begin{array}{r}
P(k+j)=P(k+j-1)+\Delta P^{s e t}(k+j-1) \\
-K_{v}(V(k+j)-V(k+j-1)) \\
V(k+j)=V(k+j-1)+s_{v} \Delta P^{s e t}(k+j-1)
\end{array}
$$

The setpoint change $\Delta P^{s e t}(k+j-1)$ is defined as the change of setpoint $P^{\text {set }}$ between two subsequent points, i.e.:

$$
\Delta P^{s e t}(k+j-1)=P^{s e t}(k+j)-P^{s e t}(k+j-1) .
$$

The value of the sensitivity $s_{v}$ takes into account the DC voltage droop gains of all VSCs and the topology of the MTDC grid. Its calculation has been detailed in [12].

At each sampling time, the above model is initialized to the latest received measurements $P^{m}(k)$ and $V^{m}(k)$, i.e.

$$
P(k)=P^{m}(k) \text { and } V(k)=V^{m}(k) .
$$

The model used for the DC grid is static, which is justified by the speed of power electronics, DC voltage and VSC controls, compared to the sampling period of the discrete controller, in the order of $250 \mathrm{~ms}$.

The prediction model can also be used in point-to-point HVDC links where the Master-Slave control logic is used instead of the droop control [25]. However, the proposed control can only be applied on the Slave VSC since the Master converter adjusts its power to control its DC voltage. In this case, the droop gain $K_{v}$ in (14) is equal to zero, and the value of $s_{v}$ accounts only for the expected DC voltage due to change of losses. 


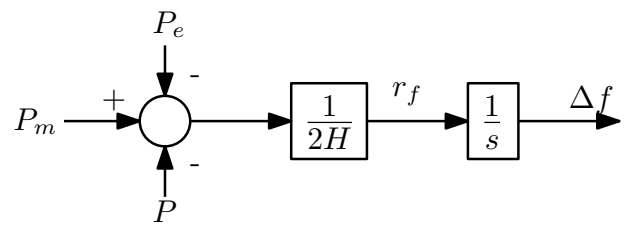

Figure 2: Simplified frequency response model

\subsection{AC frequency response model}

A model of the AC system frequency is necessary to predict a possible future violation of a frequency threshold. A simplified model is used in this work with a minimal requirement of parameters from the Transmission System Operator (TSO) of the AC system.

Figure 2 depicts the simplified frequency response model serving as a basis to derive the model used in the MPC. The following variables are involved:

- $P_{m}$ : the total mechanical power produced by the conventional power plants,

- $P_{e}$ : the total electrical power consumed in the $\mathrm{AC}$ system, produced by the generators,

- $P$ : the DC power (positive for rectifier operation) injected into the AC system (assuming only one VSC),

- $r_{f}$ : the ROCOF,

- $\Delta f$ : the frequency deviation from its nominal value,

- $H$ : the total inertia constant of the rotating masses in the AC system.

Figure 2 shows that, at every time instant:

$$
r_{f}=\frac{P_{m}-P_{e}}{2 H}-\frac{P}{2 H} .
$$

In order to derive a simplified model for inclusion in the MPC formulation the following assumptions are made:

1. $P_{m}$ and $P_{e}$ remain constant throughout the whole prediction horizon of the MPC, and equal to their values from the latest time of measurement i.e. $P_{m}(k)$ and $P_{e}(k)$. This assumption neglects the contribution of the prime movers of the power plants and the load self-regulation effect. However, by keeping the prediction horizon short enough, this approximation is easily compensated by the MPC closed-loop nature.

2. The VSC power $P$, which is adjusted by the MPC at each sampling time, has an instantaneous response and is also constant between two consecutive time instants. This is justified by the very fast response of power electronics and VSC controls, compared to the AC frequency dynamics.
Based on the aforementioned assumptions and Eq. (4), $r_{f}$ can be considered constant between two consecutive actions of the MPC. Subsequently, the frequency change between time instants $k+j$ and $k+j-1$ is given by: for $j=1, \ldots, N$ :

$$
f(k+j)-f(k+j-1)=\frac{P_{m}(k)-P_{e}(k)-P(k+j)}{2 H} T_{s}
$$

which can be decomposed as follows:

$$
\begin{aligned}
f(k+j)-f(k+j-1)= & \frac{P_{m}(k)-P_{e}(k)-P(k)}{2 H} T_{s} \\
& +\frac{P(k)-P(k+j)}{2 H} T_{s}
\end{aligned}
$$

Obviously, it is not possible to know precisely and at the rate of the controller the total mechanical and electrical powers in the $\mathrm{AC}$ system. However, the fraction in the first term in the right hand side of (6) is equal to the ROCOF at time $k$, i.e. $r_{f}(k)$, which can be measured directly by the VSC through its PLL. Consequently, $P_{m}(k)$ and $P_{e}(k)$ can be eliminated from Eq. (6) resulting in the following frequency prediction: for $j=1, \ldots, N$ :

$$
f(k+j)-f(k+j-1)=r_{f}(k) T_{s}+\frac{P(k)-P(k+j)}{2 H} T_{s}
$$

In (7) the total inertia constant $H$ is used to calculate the effect of the DC power change to the $\mathrm{AC}$ frequency. However, the exact value of this parameter might not be known. In addition, (7) assumes that only one VSC is changing its DC power to support the $\mathrm{AC}$ frequency, which might not be the case since an $\mathrm{AC}$ area can be connected to an MTDC grid with more than one VSCs. For these reasons a sensitivity $s_{f}$ is introduced. The value of $s_{f}$ requires an approximation of $H$ and should take into account the fact that more than one VSCs may be participating in frequency support.

All things considered, the AC frequency prediction model used in the MPC formulation is the following:

$$
\begin{aligned}
f(k+j)= & f(k+j-1)+r_{f}(k) T_{s} \\
& +(P(k)-P(k+j)) s_{f} T_{s}
\end{aligned}
$$

The AC frequency model is initialized to the latest frequency and ROCOF measurements $f^{m}(k)$ and $r_{f}^{m}(k)$, namely:

$$
f(k)=f^{m}(k) \text { and } r_{f}(k)=r_{f}^{m}(k) .
$$

\subsection{MPC: constrained optimization problem}

The complete quadratic programing problem at the heart of the proposed controller is described by Eqs. (9)-(16) hereafter:

$$
\begin{aligned}
& \min _{\Delta P^{\text {set }}, \epsilon, \zeta, V, P, f} \sum_{j=0}^{N_{c}-1}\left[\Delta P^{s e t}(k+j)\right]^{2} \\
& +w_{1} \sum_{j=1}^{N_{c}} \epsilon^{2}(k+j)+w_{2} \sum_{j=1}^{N_{c}} \zeta^{2}(k+j)
\end{aligned}
$$




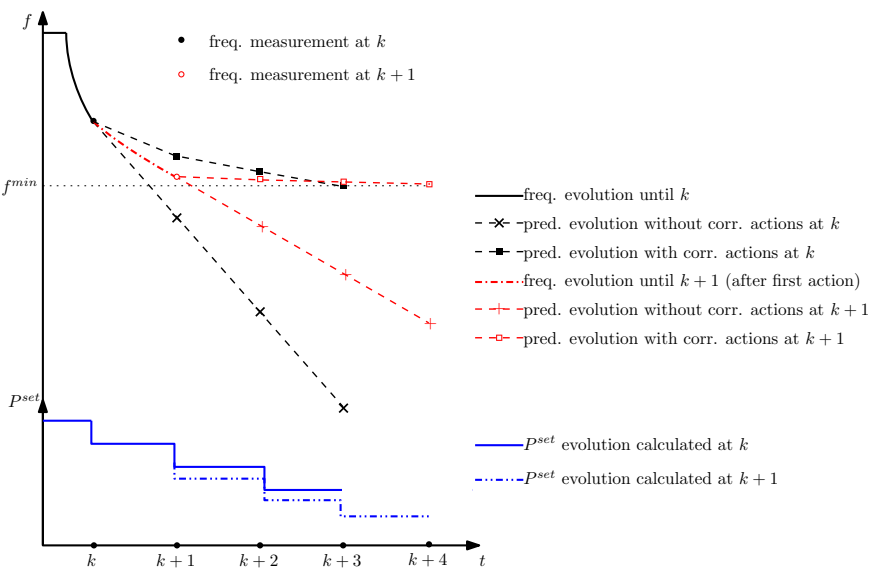

Figure 3: Illustrative example of proposed controller operation

subject to the following linear constraints, for $j=1, \ldots, N_{c}$ :

$$
\begin{gathered}
V^{\text {min }}-\epsilon(k+j) \leq V(k+j) \leq V^{\text {max }}+\epsilon(k+j) \\
P^{\text {min }} \leq P(k+j) \leq P^{\text {max }} \\
f^{\text {min }}-\zeta(k+j) \leq f(k+j) \leq f^{\text {max }}+\zeta(k+j) \\
\epsilon(k+j), \zeta(k+j) \geq 0 \\
P(k+j)=P(k+j-1)+\Delta P^{s e t}(k+j-1) \\
-K_{v}(V(k+j)-V(k+j-1)) \\
V(k+j)=V(k+j-1)+s_{v} \Delta P^{s e t}(k+j-1) \\
f(k+j)=f(k+j-1)+r_{f}(k) T_{s} \\
+[P(k)-P(k+j)] s_{f} T_{s}
\end{gathered}
$$

The first term in the objective function (9) aims to minimize the total control effort. By minimizing the $\mathcal{L}_{2}$ norm, the overall control effort is distributed throughout the whole control horizon $N_{c}$ and a smooth response is achieved. In the above formulation, the prediction horizon has been taken equal to the control horizon.

Constraint (10) specifies that the DC voltage should remain between some security limits $V^{\min }, V^{\max }$. Similarly, constraint (11) specifies that the VSC power should be between the values $P^{\min }, P^{\max }$ corresponding to the capability of the VSC. Constraint (12) keeps the AC frequency inside the limits $f^{\text {min }}$ and $f^{\max }$. Both constraints (10) and (12) can be relaxed through the positive variables $\epsilon$ and $\zeta$ in case of infeasibility. However, both $\epsilon$ and $\zeta$ are kept to small values by selecting large weighting factors $w_{1}$ and $w_{2}$ in the objective function (9). Since the DC voltage constraint is critical to avoid VSC tripping or damage, the weighting factors are chosen such that $w_{1} \gg w_{2} \gg 1$.

Equations (14)-(16) make up the prediction model as described in the previous sub-sections.

Furthermore, in case a DC voltage or frequency limit violation has been already observed, a progressive constraint tightening approach can be applied, as described in $[30]$.

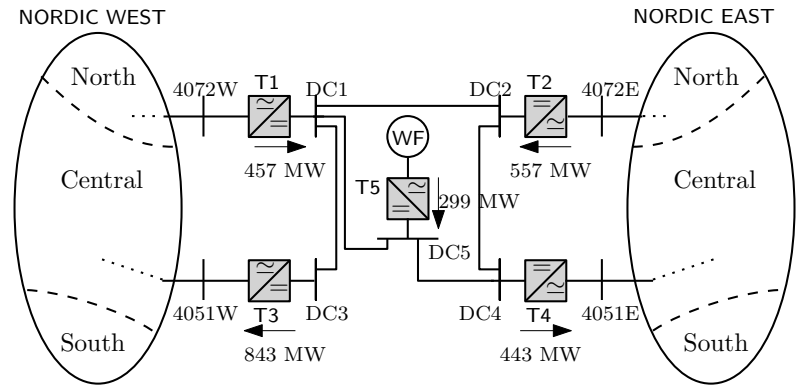

Figure 4: Test system topology and initial power flow

The controller operation for $N_{c}=3$ steps is illustrated in Fig. 3. As long as the MPC does not predict any frequency violations, the first term in the objective function ensures that no action is taken. Suppose that, at time $k$, the controller receives the new measurements and identifies that the minimum frequency limit will be violated, unless corrective actions are taken. The solution of the quadratic programming problem (9)-(16) is a sequence of control actions $\Delta P^{\text {set }}(k), \Delta P^{\text {set }}(k+1), \ldots, \Delta P^{\text {set }}\left(k+N_{c}-1\right)$ required to keep the frequency above its minimum value. The controller then applies the first action $\Delta P^{s e t}(k)$ and discards the rest of the sequence. At time $k+1$, new measurements are received and the whole procedure is repeated for the updated control horizon. As shown in Fig. 3, the updated frequency measurement at $k+1$ is different than the one predicted at time $k$ due to model simplifications, uncertainties, etc. However, the closed-loop nature of the MPC compensates for modeling errors. It has to be emphasized that the success of the VSC in keeping frequency above this limit cannot be ensured. Instead, the various constraints, in particular (11) and (10) may prevent this. Nevertheless, the proposed scheme will provide the maximum possible support to the system.

It is noted that other constraints could be also accommodated by the formulation, such as maximum power available for frequency support, maximum rate of change of frequency, maximum power requested from the other $\mathrm{AC}$ area, etc. For simplicity, the formulation has been kept to the minimal form described by Eqs. (9)-(16).

\section{Simulation results}

\subsection{Test system}

The proposed control scheme has been tested on a system consisting of two asynchronous $\mathrm{AC}$ areas and one offshore wind farm, connected through a five-terminal HVDC grid, as shown in Fig. 4. A variant of this system can be found in [31].

Each AC area is based on the Nordic test system shown in Fig. 5, set up by an IEEE Task Force and detailed in [32]. In both replicas, generator g20, which represented a large external AC system has been removed and the nearby equivalent load has been accordingly adjusted. Each 


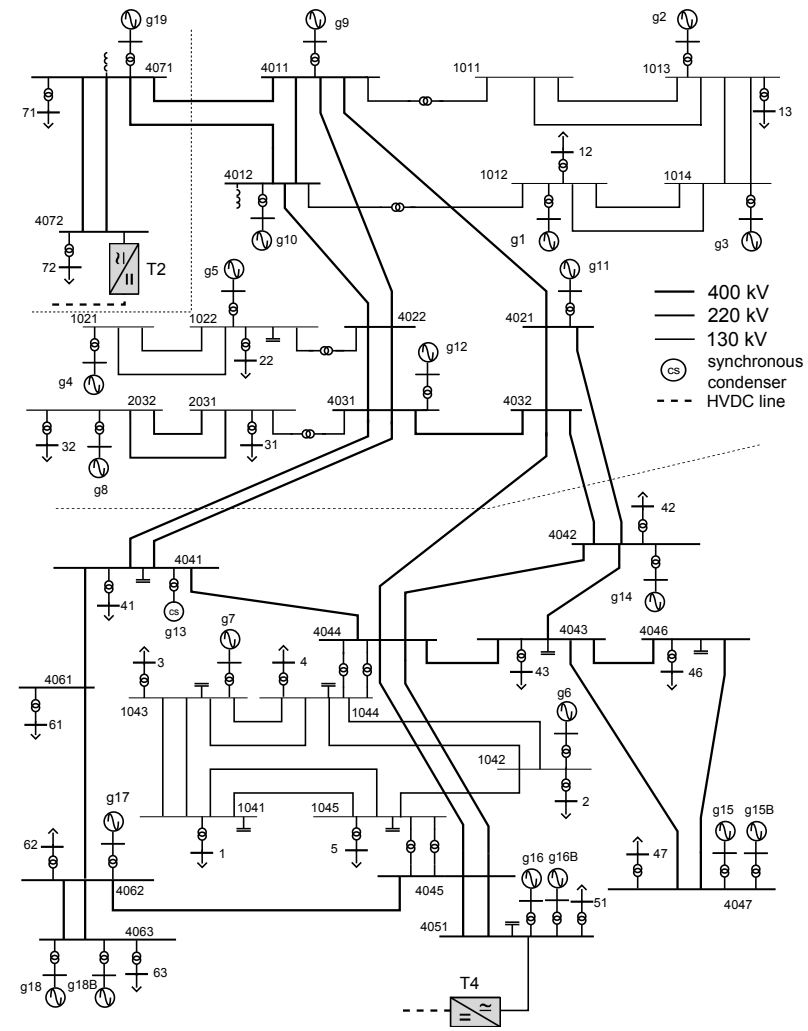

Figure 5: Nordic East test system with MTDC grid connections

subsystem has two points of connection to the MTDC grid, as shown in Fig. 4.

All generators are represented with their automatic voltage regulators, excitation systems, speed governors and turbines as detailed in [32]. Each VSC is modeled in some detail with 28 differential-algebraic equations involving the phase reactor, inner and outer control loops, PLL, filter, etc. (model of Type 6 in [33]). The DC branches are represented only by their series resistance by neglecting the series inductance and accounting for their DC capacitances in the terminal capacitors [31].

The nominal DC voltage of all VSCs is $\pm 320 \mathrm{kV}$ and the nominal power is $1000 \mathrm{MW}$. Among the five VSCs, all but T5 operate in DC voltage droop mode with $K_{v}=5 \mathrm{pu}$ (on the VSC nominal power base). The initial setpoints $P^{s e t}$ and $V^{\text {set }}$ of each VSC are set to its initial DC power and voltage. T5 imposes constant frequency and voltage on its $\mathrm{AC}$ side, thus acting as a slack bus for the offshore wind farm, merely modeled as a power injection. The initial power in each VSC is shown in Fig. 4.

All distribution buses have UFLS relays. The relays use the local frequency measurement as calculated from the bus voltage phasor measurements. If the frequency drops below a threshold for more than $100 \mathrm{~ms}$, a fraction of the total load connected to the bus is shed. The frequency thresholds and the curtailed blocks for each relay are shown in Table 1.

The disturbance considered is the tripping of generators g1 and g3 in the Nordic East area. The system re-

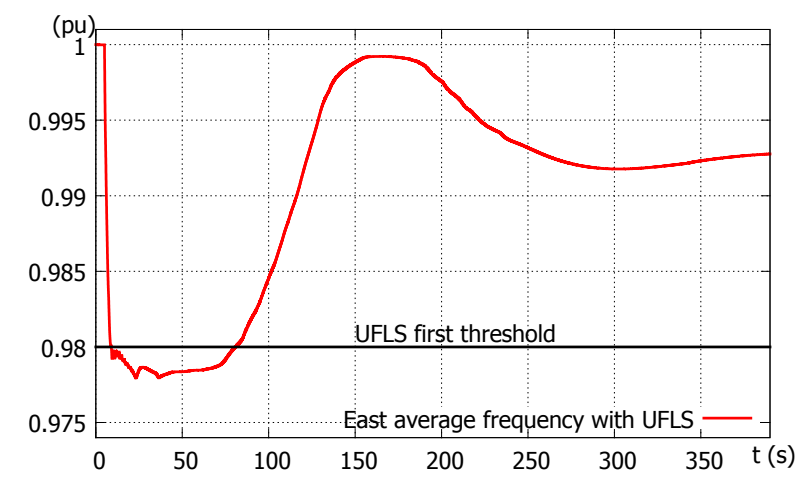

Figure 6: Average frequency of East area - Case 1

sponse is shown for the following cases:

- Case 1: the VSCs do not respond to frequency deviations, i.e. there is no support from the West area.

- Case 2: all VSCs are equipped with a traditional frequency droop control.

- Case 3: all VSCs are equipped with the proposed MPC-based control.

- Case 4: same as Case 3, but with adjusted DC voltage limits to investigate the system behavior when a DC voltage constraint becomes active.

All time simulations were performed in phasor mode with RAMSES, a simulation software developed at the University of Liège [34], using the modeling described in [35].

\subsection{Case 1: System response without frequency support}

The system is simulated without any support from the MTDC grid. The frequency evolution is shown in Fig. 6. Following the disturbance, the system frequency drops rapidly below the threshold of $0.98 \mathrm{pu}$. Consequently, all UFLS relays are triggered for the first time 2.8-4.3 s after the disturbance. Since the first shedding was not enough to arrest the frequency decline, the second level of all relays was also triggered. The total load shed is $435.9 \mathrm{MW}$, i.e. $4.5 \%$ of the total load of the system.

Following the second load shedding, the frequency of East recovers and eventually settles at around $0.993 \mathrm{pu}$ (49.65 Hz).

\subsection{Case 2: System response with frequency droop control}

The inadequacy of the simple droop control to exploit the available VSC capacity to prevent UFLS is demonstrated in this case. To this purpose, a frequency droop control is added to the control structure of all VSCs (except T5). As a result, Eq. (1) becomes:

$$
P=P^{s e t}+K_{f}(f-1)-K_{V}\left(V-V^{s e t}\right)
$$

where $K_{f}$ is the pre-defined frequency droop gain and $f$ is the frequency in per unit. This scheme has been proposed 
Table 1: UFLS relay setting

\begin{tabular}{cccc}
\hline Level & Threshold (Hz) & Threshold (pu) & \% of load shed \\
\hline 1 & 49 & 0.98 & 2.5 \\
2 & 48.9 & 0.978 & 2 \\
3 & 48.8 & 0.976 & 1 \\
4 & 48.7 & 0.974 & 1 \\
5 & 48.5 & 0.97 & 5 \\
\hline
\end{tabular}

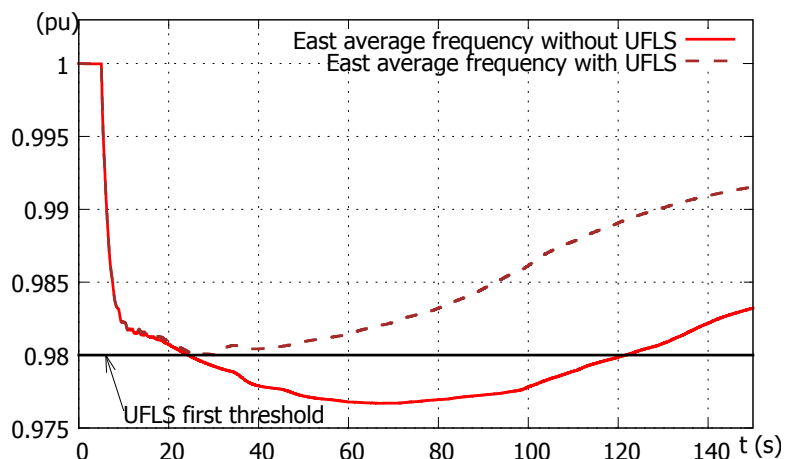

Figure 7: Frequency of East area - Case 2

in various publications, e.g. $[11,6,8]$. In the following simulation results, a typical value of 20 pu has been selected for $K_{f}$.

Figure 7 shows the frequency of the East subsystem when VSCs T2 and T4 provide support with the conventional droop control. The case without load shedding is also shown (red solid line) for comparison purposes. As in case 1 , the frequency drops quickly below the UFLS threshold and load shedding takes the necessary countermeasures. In total, 140.6 MW of load are shed, at 14 buses. Following the load shedding, the East frequency recovers above $0.99 \mathrm{pu}$.

The DC powers of VSCs T1-T4 are shown in Fig. 8. As expected, the powers of $\mathrm{T} 2$ and $\mathrm{T} 4$ decrease following the disturbance (i.e. more power is injected into the East subsystem). The powers of T1 and T3 increase due to DC voltage droop control to restore the power balance in the MTDC grid and stabilize the DC voltages. Following the load shedding after $t=20 \mathrm{~s}$ and the subsequent restoration of the East frequency, all VSC powers partially return to their initial values.

The DC voltages are shown in Fig. 9. The demand of power from the East system leads to a drop of the overall DC voltage level. The voltages follow the response of the East frequency and recover following the UFLS.

\subsection{Case 3: System response with MPC-based control}

In this case, all VSCs (except T5) are equipped instead with the proposed frequency support scheme.

The DC voltage limits have been chosen equal to $V^{\text {min }}=$ 0.90 and $V^{\max }=1.10$. The active power limits of each

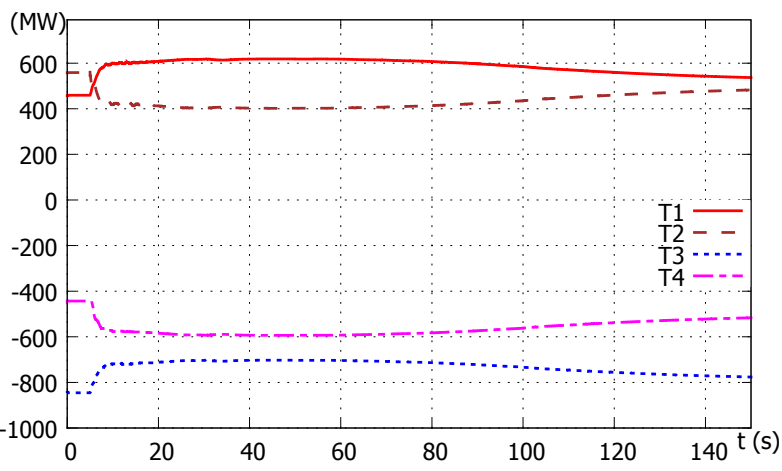

Figure 8: VSC DC powers - Case 2

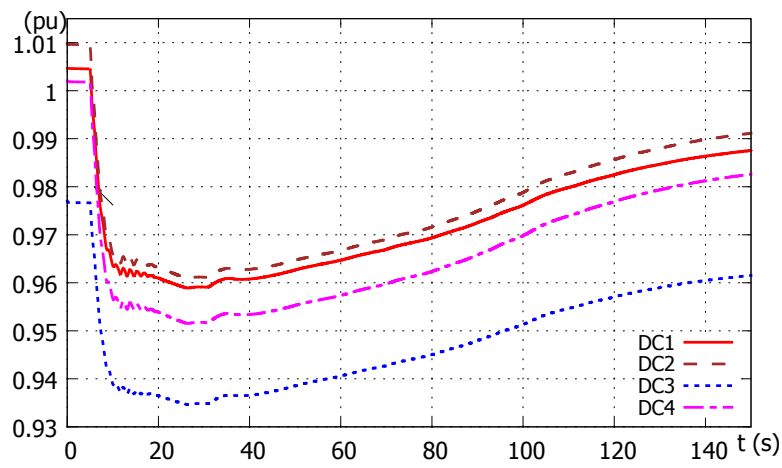

Figure 9: MTDC grid DC voltages - Case 2

VSC have been set equal to the VSC nominal active power i.e. $P^{\min }=-10$ and $P^{\max }=+10 \mathrm{pu}$ on a $100 \mathrm{MW}$ base. The weighting factors $w_{1}$ and $w_{2}$ have been selected equal to $10^{3}$ and $10^{6}$, respectively.

The frequency limits are set to $f^{\text {min }}=0.985 \mathrm{pu}$ and $f^{\max }=1.015 \mathrm{pu}$ (see $\left.(12)\right)$. In fact, following large disturbances, the local frequencies are exposed to electromechanical oscillations, which could trigger UFLS in some locations. Therefore, the lower frequency limit $f^{\text {min }}$ has been selected slightly higher than the UFLS relays threshold to prevent inadvertent load shedding.

All discrete controllers have a control horizon of $N_{c}=4$ steps and a sampling time $T_{s}=0.25 \mathrm{~s}$, which is long enough compared to the time constants of power electronics but short with respect to frequency dynamics. In order to synchronize the VSCs acting on the same AC area, the controls $\Delta P^{s e t}$ are applied at discrete times $k T_{s}$ $(k=1,2, \ldots)$, assuming that each controller is relying on a 


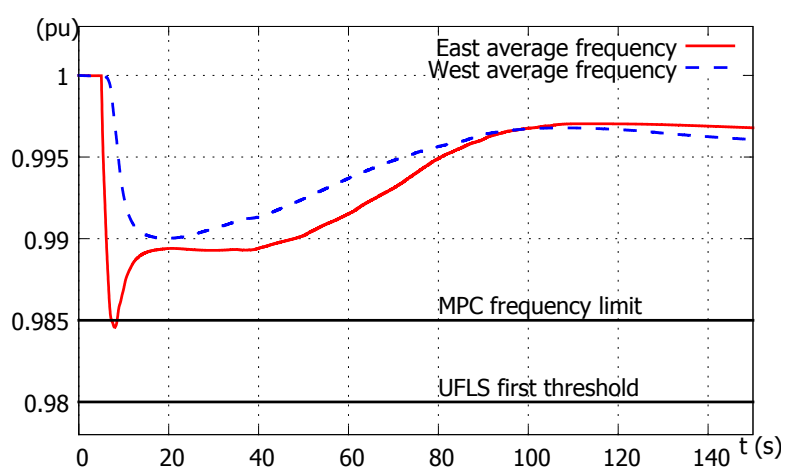

Figure 10: Frequency of East area - Case 3

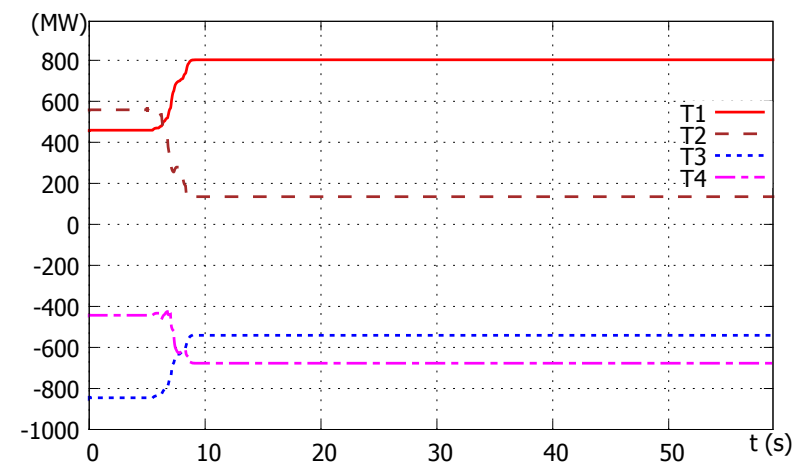

Figure 11: VSC DC powers - Case 3

GPS-synchronized clock. Each VSC collects the measurements $P^{m}(k), V^{m}(k), f^{m}(k)$ and $r_{f}^{m}(k)$ at times $k T_{s}-0.05$ $\mathrm{s}(k=1,2, \ldots)$ to account for the time needed to solve the optimization problem.

The system response is shown in Figs. 10-12. To begin with, Fig. 10 shows that the proposed controller successfully manages to keep the East frequency above the $f^{\text {min }}$ threshold, with just a small violation, which can be attributed to the approximations embedded in the formulation. Nevertheless, load shedding has been successfully prevented by the fast support from the MTDC grid. Obviously, this increased demand is reflected on the West system frequency which experiences a larger drop than with the conventional frequency droop control. However, this drop is acceptable and does not cause any problem in the West area. Furthermore, the selectivity of the proposed scheme has been demonstrated since the MPC controllers of T1 and $\mathrm{T} 3 \mathrm{did}$ not react to the frequency drop in the West system.

Figure 11 displays the DC powers of VSCs T1-T4. Following the disturbance, the powers of T2 and T4 quickly decrease to support the AC system. As soon as the frequency starts rising, the controllers identify that enough power has been injected in their $\mathrm{AC}$ grid and they stop adjusting the power setpoints of the VSCs. It is interesting to note that $\mathrm{T} 2$ provides slightly more power than T4. This is due to electromechanical oscillations affecting the local measurement of the ROCOF, especially the fre-

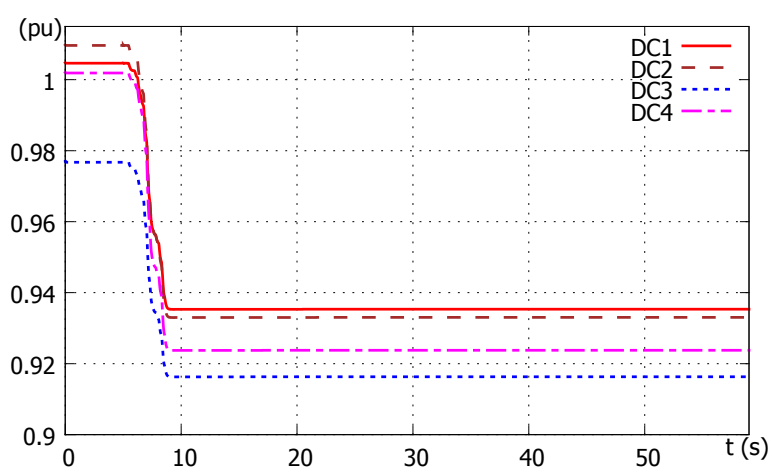

Figure 12: MTDC grid DC voltages - Case 3

quency sensed by T2, which is closer to the disturbance. This difference can be reduced by filtering these measurements.

The DC voltages are shown in Fig. 12, where it can be seen that they decrease by the actions of the controller, eventually settling at a value above the lower limit $V^{\text {min }}$ involved in the MPC formulation.

It is important to note that, in contrast to Case 2, there is no restoration of the VSC power following the AC frequency restoration. The reason is that the frequency is inside the MPC allowed range and the controller does not take control actions anymore, as already shown in Fig. 11. In addition, due to the absence of any restoration of VSC power, the steady-state frequency of the East area (where the initial contingency occurred) is higher than the steadystate frequency of the West area. These "drawbacks" of the proposed control method can be easily resolved at a slower time frame by a centralized control of the MTDC grid, as the one proposed in [30].

\subsection{Case 4: Effect of DC voltage constraints}

This last case demonstrates the effect of activating a DC voltage constraint while in progress of frequency support.

If the DC voltage of the controller violates its limit, the term corresponding to $\epsilon$ in the objective function (9) becomes dominant due to the large value selected for the weighting factor $w_{1}$. Consequently, the MPC switches to keeping the DC voltage above its limit, which is equivalent to switching the VSC to DC voltage control. Obviously, frequency support becomes less effective as long as the DC voltage of the VSC is at (or below) its limit. Furthermore, the DC voltages are not uniform throughout the MTDC grid, but depend on the MTDC grid topology and the power flows, in contrast to frequency in $\mathrm{AC}$ systems. Therefore, assuming that more than one VSCs have been equipped with the proposed controller with the same limits $V^{\min }, V^{\max }$, it is reasonable that this constraint may become active in one VSC (not known a priori) before the others.

Coming back to the test system of Fig. 4 two cases can be envisaged: (i) one of $\mathrm{T} 1$ or $\mathrm{T} 3$ reaches first its $V^{\text {min }}$ 


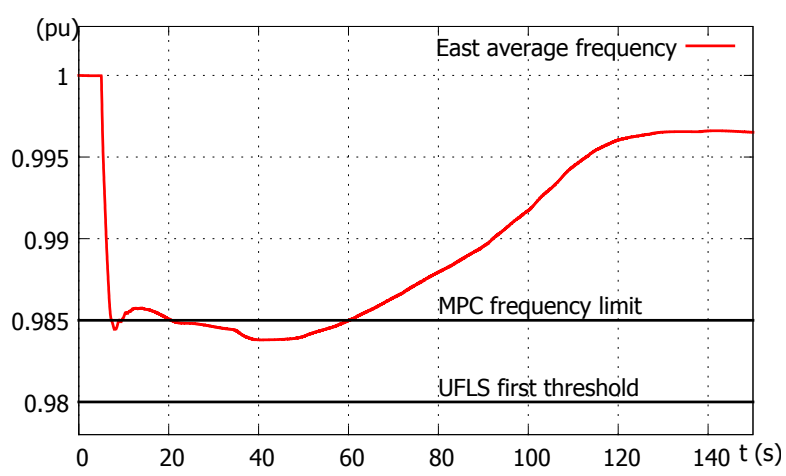

Figure 13: Frequency of East area - Case 4

limit while providing the power requested by $\mathrm{T} 2$ and $\mathrm{T} 4$, and (ii) one of $\mathrm{T} 2$ or $\mathrm{T} 4$ reaches first its $V^{\text {min }}$ limit. Case (i) would only affect the sharing between T1 and T3 of the total power requested by $\mathrm{T} 2$ and $\mathrm{T} 4$. Therefore, the effect to the frequency support to the East subsystem would be negligible, and this case is not further detailed. On the other hand, case (ii) could have a significant impact on the overall frequency support. To demonstrate this, the voltage limits of VSCs T2 and T4 only have been set to the more constraining values of $V^{\min }=0.95$ and $V^{\max }=1.05$ $\mathrm{pu}$.

The frequency in the East system is shown in Fig. 13. Initially, the frequency behavior is similar to the one of Fig. 10 with only a small violation of the threshold of $0.985 \mathrm{pu}$. However, the frequency starts declining again and drops below this threshold until approximately $60 \mathrm{~s}$ where it slowly recovers above this threshold. The cause for this second frequency decline is shown in Figs. 14 and 15, showing the VSC DC powers and MTDC grid voltages, respectively. Following the activation of frequency support by $\mathrm{T} 2$ and T4, their DC voltages are quickly depressed with T4 dropping below its limit. Therefore, T4 starts drawing power to smoothly restore the DC voltage at its limit, leading to the second frequency decline. Meanwhile, the DC voltage of T2 is still above its limit; hence, when the frequency drops again below the threshold, T2 starts injecting more power in the AC grid. However, this power is provided mainly by $\mathrm{T} 4$, whose behavior has become similar to that of a master VSC, leading to a slow shift of power from T4 to T2. This power shift stops when the voltage of $\mathrm{T} 2$ also reaches its minimum value. Clearly, this situation arises since no communication is utilized to inform VSCs that the behavior of the MTDC grid voltages has significantly been altered. However, as mentioned in [12] where a similar shift of power was observed, the use of a slower centralized controller could restore the DC voltage near its nominal value, thus "unblocking" the VSC whose voltage constraint is active. This in turn would enable to resume the frequency support. Finally, it should be noted that even in this case, UFLS has been successfully prevented.

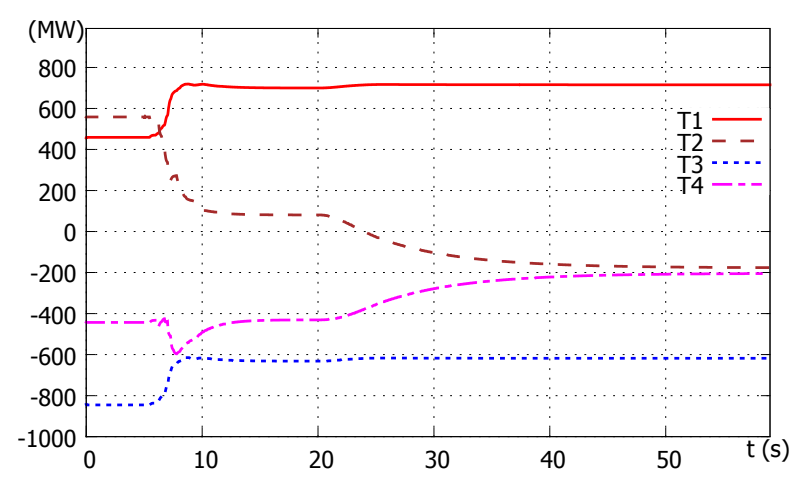

Figure 14: VSC DC powers - Case 4

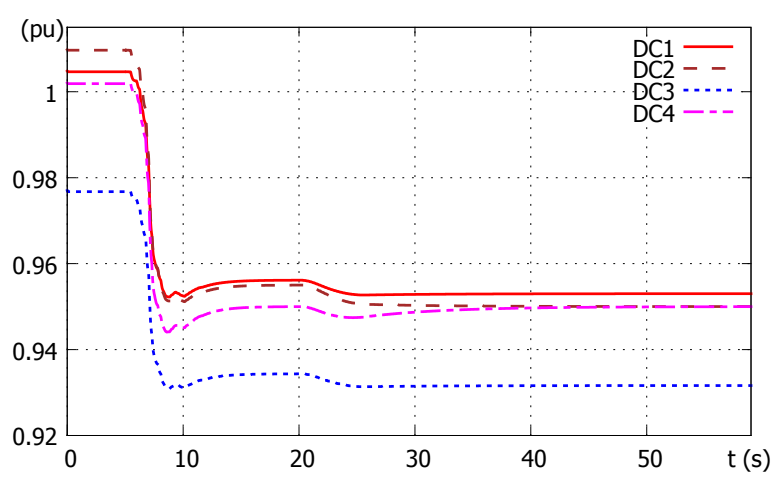

Figure 15: MTDC grid DC voltages - Case 4

\section{Summary and future extensions}

\subsection{Summary}

This paper has presented a novel method to support the frequency of asynchronous AC systems connected to an MTDC grid or through a point-to-point HVDC link. Instead of defining a desired participation to primary frequency control, the proposed scheme is inspired of MPC and adjusts the power of the VSCs within its available limits to contain the $\mathrm{AC}$ frequency between acceptable values, thereby decreasing or avoiding load curtailment.

The controller is normally inactive and acts when an emergency condition is detected. The detection is made with the use of a simplified AC frequency dynamics model, which requires only minimal information about the $\mathrm{AC}$ system. In addition, DC voltage constraints have been included in the formulation to ensure the integrity of the MTDC grid.

Simulation results on a test system have demonstrated the effectiveness of the control scheme and its better performance compared to the conventional frequency droop control. In addition, the behavior of the proposed controller when DC voltage constraints prevent frequency support has been described.

\subsection{Future extensions}

Future research will address the controller behavior following DC grid contingencies, mainly the outage of a VSC, 
which could cause a frequency emergency in all AC areas connected to the MTDC grid.

The next steps will be:

- the investigation of the possible benefits from using a more accurate frequency prediction model,

- the coordination of the proposed controller with a centralized control scheme of the MTDC grid aimed at restoring the powers of the VSC to their original values,

- the incorporation of the proposed method in the security analysis of an AC grid.

\section{References}

[1] D. Van Hertem, M. Ghandhari, Multi-terminal VSC HVDC for the European supergrid: Obstacles, Renewable and Sustainable Energy Reviews 14 (2010) 3156-3163.

[2] T. K. Vrana, System Design and Balancing Control of the North Sea Super Grid, Ph.D. thesis, Norwegian Univ. of Sc. and Techn., 2013.

[3] D. Van Hertem, R. H. Renner, Ancillary services in electric power systems with HVDC grids, IET Generation, Transmission \& Distribution 9 (2015) 1179-1185.

[4] G. Lalor, A. Mullane, M. O'Malley, Frequency Control and Wind Turbine Technologies, IEEE Trans. on Power Systems 20 (2005) 1905-1913.

[5] J. Fradley, R. Preece, M. Barnes, VSC-HVDC for Frequency Support (a review), 13th IET International Conference on AC and DC Power Transmission (ACDC 2017) 2 (2017).

[6] T. M. Haileselassie, K. Uhlen, Primary frequency control of remote grids connected by multi-terminal HVDC, in: Proc. IEEE PES General Meeting 2010.

[7] N. R. Chaudhuri, R. Majumder, B. Chaudhuri, System Frequency Support Through Multi-Terminal DC (MTDC) Grids, IEEE Trans. on Power Systems 28 (2013) 347-356.

[8] R. Wiget, G. Andersson, M. Andreasson, D. V. Dimarogonas, K. H. Johansson, Dynamic simulation of a combined AC and MTDC grid with decentralized controllers to share primary frequency control reserves, in: Proc. IEEE PES Eindhoven PowerTech 2015.

[9] C. E. Spallarossa, Y. Pipelzadeh, T. C. Green, Influence of frequency-droop supplementary control on disturbance propagation through VSC HVDC links, in: Proc. IEEE PES General Meeting 2013.

[10] O. D. Adeuyi, M. Cheah-Mane, J. Liang, N. Jenkins, Y. Wu, C. Li, X. Wu, Frequency support from modular multilevel converter based multi-terminal HVDC schemes, in: Proc. IEEE PES General Meeting 2015.

[11] S. Akkari, J. Dai, M. Petit, X. Guillaud, Coupling between the frequency droop and the voltage droop of an $\mathrm{AC} / \mathrm{DC}$ converter in an MTDC system, in: Proc. 2015 IEEE PES Eindhoven PowerTech.

[12] L. Papangelis, M.-S. Debry, P. Panciatici, T. Van Cutsem, A receding horizon approach to incorporate frequency support into the AC/DC converters of a multi-terminal DC grid, Electric Power Systems Research 148 (2017).

[13] L. Papangelis, M.-S. Debry, T. Van Cutsem, P. Panciatici, Local control of $\mathrm{AC} / \mathrm{DC}$ converters for frequency support between asynchronous AC areas, in: Proc. IEEE Manchester PowerTech 2017.

[14] Y. Phulpin, Communication-Free Inertia and Frequency Control for Wind Generators Connected by an HVDC-Link, IEEE Trans. on Power Systems 27 (2012) 1136-1137.

[15] Y. Pipelzadeh, B. Chaudhuri, T. C. Green, Inertial response from remote offshore wind farms connected through VSCHVDC links: A Communication-less scheme, in: Proc. IEEE PES General Meeting 2012.
[16] S. I. Nanou, G. N. Patsakis, S. A. Papathanassiou, Assessment of communication-independent grid code compatibility solutions for VSC-HVDC connected offshore wind farms, Electric Power Systems Research 121 (2015) 38-51.

[17] B. Silva, C. L. Moreira, L. Seca, Y. Phulpin, J. a. Pecas Lopes, Provision of Inertial and Primary Frequency Control Services Using Offshore Multiterminal HVDC Networks, IEEE Trans. on Sustainable Energy 3 (2012) 800-808.

[18] C. Feltes, I. Erlich, Variable Frequency Operation of DFIG based Wind Farms connected to the Grid through VSC-HVDC Link, in: Proc. IEEE PES General Meeting 2007.

[19] I. Martinez-Sanz, Control of AC / DC Systems for Improved Transient Stability and Frequency Support Provision, Ph.D. thesis, Imperial College London, 2015.

[20] J. Zhu, C. D. Booth, G. P. Adam, A. J. Roscoe, C. G. Bright, Inertia Emulation Control Strategy for VSC-HVDC Transmission Systems, IEEE Trans. on Power Systems 28 (2013) 1277-1287.

[21] D. Jing, Y. Phulpin, A. Sarlette, D. Ernst, Voltage control in an HVDC system to share primary frequency reserves between nonsynchronous areas, in: Proc. 17th Power Systems Computation Conference 2011.

[22] L. Bizumic, R. Cherkaoui, U. Hger, Ch.17 - interface Protection, in: Monitoring, Control and Protection of Interconnected Power Systems, Springer, 2014, pp. 333-347.

[23] J. M. Maciejowski, Predictive control: with constraints, Pearson education, 2002.

[24] S. J. Qin, T. A. Badgwell, A survey of industrial model predictive control technology, Control Engineering Practice 11 (2003) 733-764.

[25] P. Rault, Dynamic Modeling and Control of Multi-Terminal HVDC Grids, Ph.D. thesis, Ecole Centrale de Lille, L2EP, 2014.

[26] C. Dierckxsens, K. Srivastava, M. Reza, S. Cole, J. Beerten, R. Belmans, A distributed DC voltage control method for VSC MTDC systems, Electric Power Systems Research 82 (2012) $54-58$.

[27] F. Thams, S. Chatzivasileiadis, E. Prieto-araujo, R. Eriksson, Disturbance Attenuation of DC Voltage Droop Control Structures in a Multi-Terminal HVDC Grid, in: Proc. IEEE Manchester PowerTech 2017.

[28] J. Beerten, R. Belmans, R. Eriksson, Influence of DC voltage droop settings on AC system stability, in: 10th IET International Conference on AC and DC Power Transmission (ACDC 2012).

[29] ENTSO-E, Technical background for the Low Frequency Demand Disconnection requirements, 2014.

[30] L. Papangelis, M.-S. Debry, P. Panciatici, T. Van Cutsem, Coordinated Supervisory Control of Multi-Terminal HVDC Grids: a Model Predictive Control Approach, IEEE Trans. on Power Systems (2017).

[31] L. Papangelis, X. Guillaud, T. Van Cutsem, Frequency support among asynchronous AC systems through VSCs emulating power plants, in: Proc. 2015 11th IET Intern.l Conf. on AC and DC Power Transmission.

[32] IEEE PES Task Force, Test Systems for Voltage Stability Analysis and Security Assessment, 2015. Technical Report PES-TR19, Available online on IEEE PES Resource Center. http://resourcecenter.ieee-pes.org/pes/product/technicalpublications/PESTR19.

[33] CIGRE WG B4.57, Guide for the Development of Models for HVDC Converters in a HVDC Grid, 2014. CIGRE Technical Brochure 604.

[34] P. Aristidou, D. Fabozzi, T. Van Cutsem, Dynamic Simulation of Large-Scale Power Systems Using a Parallel SchurComplement-Based Decomposition Method, IEEE Trans. on Parallel and Distributed Systems 25 (2014) 2561-2570.

[35] P. Aristidou, L. Papangelis, X. Guillaud, T. Van Cutsem, Modular modelling of combined AC and DC systems in dynamic simulations, in: Proc. IEEE Eindhoven PowerTech 2015. 